\title{
Eradication of therapy-resistant cancer cells in gastrointestinal organs (Review)
}

\author{
SHIMPEI NISHIKAWA ${ }^{1,2}$, MASAMITSU KONNO ${ }^{1}$, ATSUSHI HAMABE ${ }^{1,2}$, SHINICHIRO HASEGAWA $^{1,2}$, \\ HISATAKA OGAWA ${ }^{1,2}$, YOSHIHIRO KANO ${ }^{1,2}$, TAKAHITO FUKUSUMI ${ }^{1,3}$, KATSUYA OHTA $^{1,2}$, YUKO NOGUCHI ${ }^{1}$, \\ MIYUKI OZAKI $^{1,2}$, TOSHIHIRO KUDO ${ }^{1}$, DAISUKE SAKAI ${ }^{1}$, NAOTSUGU HARAGUCHI ${ }^{1}$, TAROH SATOH ${ }^{1}$, \\ YUICHIRO DOKI ${ }^{2}$, MASAKI MORI ${ }^{2}$ and HIDESHI ISHII ${ }^{1}$
}

Departments of ${ }^{1}$ Frontier Science for Cancer and Chemotherapy, ${ }^{2}$ Gastroenterological Surgery and ${ }^{3}$ Otorhinolaryngology-

Head and Neck Surgery, Osaka University, Graduate School of Medicine, Suita, Osaka 565-0871, Japan

Received June 9, 2012; Accepted July 17, 2012

DOI: $10.3892 / \mathrm{mco} .2012 .13$

\begin{abstract}
Early stages of cancer are curable by surgical removal of the primary lesions, however, more advanced cases are often refractory to therapeutic approaches and are more commonly life-threatening, primarily due to cancer metastasis in gastrointestinal cancers. Such biological events are collectively characterized as tumor heterogeneity, the cause of which is the existence of cancer stem cells. To improve cancer survival, therapy-resistant cancer cells should be eradicated. To this end, recent rapid progress in medical science, such as innovative medical technologies including cancer reprogramming, RNA pharmacology and drug delivery systems, all of which effectively target cancer stem cells, has facilitated this objective.
\end{abstract}

\section{Contents}

1. Introduction

2. Eradication of chemoradiation therapy-resistant cancer cells

3. RNA pharmacology

4. DDS for CSCs

5. Conclusions

Correspondence to: Dr Masaki Mori, Department of Gastroenterological Surgery, Osaka University, Graduate School of Medicine, Suita, Yamadaoka 2-2, Osaka 565-0871, Japan

E-mail: mmori@gesurg.med.osaka-u.ac.jp

Dr Hideshi Ishii, Department of Frontier Science for Cancer and Chemotherapy, Osaka University, Graduate School of Medicine, Suita, Osaka 565-0871, Japan

E-mail: hishii@gesurg.med.osaka-u.ac.jp

Key words: cancer therapy, cancer stem cell, gastrointestinal organs

\section{Introduction}

Cancer remains an unresolved issue in the field of biology. Although early stages of cancer are curable by surgical treatment, removal of deleterious cancer cells by surgery in advanced stages remains a challenge. Furthermore, advanced cancer is occasionally refractory even to chemoradiation due to the quantity and quality of malignant cells in the body, i.e., the development of therapy-resistant cancer cells in the background of the genetic instability of cancer $(1,2)$. To modify the malignant phenotype of various types of cancer, a novel innovative technology of cancer reprogramming that is achievable through RNA pharmacology has been developed. This technology may be beneficial for reversing the therapeutic resistance of gastrointestinal cancer in combination with an efficient drug delivery system (DDS).

\section{Eradication of chemoradiation therapy-resistant cancer cells}

Although cancer is a genetic and epigenetic disorder, epigenetic regulation plays a critical role in the malignant phenotype of various types of cancer $(1,2)$. Given that defined factors induce reprogramming, such as the development of induced pluripotent stem cells (iPSCs) via the introduction of defined biological factors including Oct4 (also known as Pou5f1), Sox2, Klf4 and c-Myc in mouse (3) and human fibroblasts (4) through an epigenetic-based mechanism (5), previous data indicated that retrovirus-mediated defined factor gene transfer in gastrointestinal cancer cells resulted in the induction of ES-like gene and protein expression (patterns induced from the endoderm of the gastrointestinal tract to the mesoderm and ectoderm) (6). Notably, the retrovirus-mediated exogenous expression of Oct $4 /$ Sox $2 /$ Klf4/Myc or Oct $4 /$ Sox $2 /$ Klf4 sensitized gastrointestinal cancer cells to vitamins and other chemotherapeutic agents (6). The reprogrammed cells exhibited molecular changes in DNA methylation and histone modification, and the epigenome resembled that of embryonic stem cells. The promoter region of p16/INK4A was demethylated, resembling the heavily demethylated parental state (6). 
Although in vivo experiments involving short-term-cultured reprogrammed cells showed an inhibition of tumorigenicity in DLD-1 colorectal cancer cells (6), long-term-cultured reprogrammed cells with gain-of-function mutations, including $\mathrm{TP} 3 \mathrm{R}^{175 \mathrm{H}}$ and KRAS ${ }^{\mathrm{G} 12 \mathrm{D}}$, elicit a malignant transformation with the activation of c-Myc in K-ras- and TP53-mutated HuCC-T1 cholangiocellular carcinoma cells, suggesting a role of such oncogenic mutations in the reactivation of a malignant phenotype (7). Previous studies on the role of TP53 in reprogramming have demonstrated that decreasing the expression of TP53 enables the development of murine fibroblasts in iPSCs capable of generating germline-transmitting chimeric mice, indicating that TP53 may not be necessary for reprogramming. Instead, silencing TP53 is likely to significantly increase the reprogramming efficiency of human somatic cells (8-10). Additionally, gain-of-function TP53 oncogenic mutations enhance defined factor-mediated cell reprogramming (11), suggesting that the mutation context of TP53 is affected by the quality and quantity of reprogramming events. Reprogramming efficiency was increased in hypoxia (12), an effect that was observed in cancer cells (13). Taken together, basic research indicates that tumor suppressor pathways are involved in the regulation of cellular reprogramming, and modification of these pathways is likely to lead to the sensitization of cancer cells to currently used chemoradiation therapies. Therefore, although currently used chemoradiation therapies potentially induce resistance, cellular reprogramming in combination with chemoradiation therapies would modify the cell nature and overcome therapeutic resistance, allowing the eradication of therapy-resistant cancer cells.

\section{RNA pharmacology}

The aforementioned cellular reprogramming is based on viral-mediated gene transfers; thus, genomic insertion requires attention during clinical application. Findings of recent studies demonstrated that human and mouse somatic cells can be reprogrammed into iPSCs through the forced expression of miRNAs, completely eliminating the need for ectopic protein expression $(14,15)$. Anokye-Danso et al (14) revealed that the lentiviral-mediated transfection of immature miR302/367 sequences generated reprogrammed cells (miR302/367 iPSCs) with similar characteristics to Oct4/Sox $/ \mathrm{Klf} 4 / \mathrm{Myc}$ iPSCs, including pluripotency marker expression and teratoma formation, and for mouse cells, chimera and germline contribution. miR367 expression is required for miR302/367-mediated reprogramming, since it activates Oct4 gene expression, as is Hdac2 suppression (14). Conversely, the direct transfection of mature double-stranded miRNAs (a combination of the miR200c, miR-302s and miR-369s family sequences) resulted in the generation of iPSCs from differentiated adipose-derived stem cells in humans and mice (15). This reprogramming method does not require vector-based gene transfer and, thus, holds significant potential in biomedical research and regenerative medicine. The introduction of these factors is likely to be beneficial for medical application because the RNAs can be chemically synthesized and should be free of genomic insertions, which are able to cause troublesome genomic damage. Eventually, the introduction of these miRNAs may modify cancer malignancies (submitted data) (16).
miR-302 transfection induces ES-like phenotypes of skin cancer (17). miR-302 also inhibits tumorigenicity via the coordinated suppression of the CDK2 and CDK4/6 cell cycle pathways (16). In a study by Lin et al, concurrent silencing of BMI-1, a cancer stem cell (CSC) marker targeted by miR-302, was found to promote the tumor-suppressor functions of p16INK4a and p14/p19Arf directed against CDK4/6-mediated cell proliferation. miR-302 inhibits human pluripotent stem cell tumorigenicity by enhancing multiple $\mathrm{G}_{1}$ phase-arrest pathways (16). Results of another study on glioma indicated that the miR-302-367 cluster markedly affects the self-renewal and infiltration properties of glioma-initiating cells through CXCR4 repression and the consequent disruption of the SHH-GLI-NANOG network (18). Thus, the miR-302/367 cluster is able to trigger a cascade of inhibitory events that efficiently lead to the disruption of CSC-like and tumorigenic properties (18).

\section{DDS for CSCs}

Tumors are characterized by heterogeneous cell populations harboring distinct functional roles in terms of their tumor formation, metastasis and drug resistance abilities (19). The clonal evolution model suggests that most tumor cells harbor the ability to self renew and maintain tumor growth, whereas the CSC model places a unique cancer cell that has self-renewing and differentiation potential at the apex of the hierarchy $(20,21)$. Evidence for the existence of tumor cells with stem cell-like properties has shed light on the field of cancer research since its discovery in a study on acute myeloid leukemia (22). This concept has been applied to both hematopoietic malignancy and solid tumors, such as cancers of the head and neck (23), gastrointestinal system (24), colon $(25,26)$, breast $(27)$ and brain $(28,29)$. CSCs are defined as cells with indefinite tumor-reconstituting potential that drive the formation and fuel the growth of tumors (21). Given the similarity between normal stem cells and CSCs in that a distinct small population can reconstitute tumors when isolated from tumor tissues and can be inoculated into an immunodeficient animal model, CSCs are characterized concomitantly with normal stem cells. Normal stem cells are characterized as being able to self-renew, potentially divide and differentiate to generate all functional elements of a particular tissue, as well as to stringently regulate stem cell numbers $(30,31)$. CSCs are defined as cells within tumors with tumor-reconstituting potential $(21,31)$, as demonstrated by their inoculation into immunodeficient animals, which has no control over cell numbers in serial transplantations (31).

DDSs are designed to enhance the pharmacological and therapeutic effects of drugs (32). Problems impeding the application of particulate DDSs have been resolved, and several DDS formulations, such as nanoparticles, have been approved for clinical use as anticancer therapies. Nanoparticles encapsulate conventional anticancer drugs and new genetic drugs, such as RNA-based drugs, and efficiently release them in low $\mathrm{pH}$ areas such as the acidic environment in lysosomes, thus enhancing the targeting effect in the cytoplasm. Targeting CSCs via DDSs should be useful for maximizing the anti-tumorigenic effect and minimizing the off-target toxicity of drugs. A recent study indicated that vitronectin is a candidate extrinsic inducer 
of CSC differentiation and tumor formation (33). Findings of that study demonstrated that blocking integrin $\alpha \mathrm{V} \beta 3$ inhibits differentiation and subsequently tumor formation, and CSCs must be engaged by one or more extracellular signals to differentiate and initiate tumor formation, thereby defining a new axis for future novel therapies aimed at extrinsic and intracellular pathways (33). Thus, CSC-specific surface molecules are candidate targets for CSC-directed drug delivery and controlling integrin-dependent adhesion and motility as well as the cell phenotype and induction of anti-tumorigenic effects (34). The identification of reliable markers that specifically mark the CSC populations in a particular malignancy and reflect their phenotypic characteristics for use in a clinical setting and for predicting patient outcomes remains a challenge. Although several CSC-targeted therapies have demonstrated considerable promise in eradicating CSC populations, the confirmation of their efficacy in clinical settings remains to be established.

\section{Conclusions}

The present review discussed novel cancer treatment strategies for eradicating therapy-resistant cancer cells. These strategies include i) the reprogramming of cancer cells to modify the malignant nature of tumor cells, ii) RNA pharmacology to develop novel medicines and iii) the efficient targeting of CSCs using DDSs. Targeting therapy-resistant CSCs is a breakthrough in both the understanding of malignant behavior and the development of new therapeutic approaches. Elucidating the regulation of the CSC molecular network through various epigenetic mechanisms, studies of which are now in progress, is likely to become the future trend of research and provide valuable contributions to the investigation of CSCs.

\section{References}

1. Hanahan D and Weinberg RA: The hallmarks of cancer. Cell 100: 57-70, 2000.

2. Hanahan D and Weinberg RA: Hallmarks of cancer: the next generation. Cell 144: 646-674, 2011.

3. Takahashi K and Yamanaka S: Induction of pluripotent stem cells from mouse embryonic and adult fibroblast cultures by defined factors. Cell 126: 663-676, 2006.

4. Takahashi K, Tanabe K, Ohnuki M, et al: Induction of pluripotent stem cells from adult human fibroblasts by defined factors. Cell 131: 861-872, 2007.

5. Yamanaka S: Elite and stochastic models for induced pluripotent stem cell generation. Nature 460: 49-52, 2009.

6. Miyoshi N, Ishii H, Nagai K, et al: Defined factors induce reprogramming of gastrointestinal cancer cells. Proc Natl Acad Sci USA 107: 40-45, 2010.

7. Nagai K-i, Ishii H, Miyoshi N, et al: Long-term culture following ES-like gene-induced reprogramming elicits an aggressive phenotype in mutated cholangiocellular carcinoma cells. Biochem Biophysic Res Commun 395: 258-263, 2010.

8. Zhao Y, Yin X, Qin H, et al: Two supporting factors greatly improve the efficiency of human iPSC generation. Cell Stem Cell 3: 475-479, 2008.

9. Kawamura T, Suzuki J, Wang YV, et al: Linking the p53 tumour suppressor pathway to somatic cell reprogramming. Nature 460 1140-1144, 2009

10. Hong H, Takahashi K, Ichisaka T, et al: Suppression of induced pluripotent stem cell generation by the p53-p21 pathway. Nature 460: 1132-1135, 2009.

11. Moon JH, Ishii H, Dewi DL, et al: Gain-of-function oncogenic mutations in TP53 enhance defined factor-mediated cellular reprogramming. Nat Preced: 2011. doi:10.1038/npre.2011.5681.1.
12. Yoshida Y, Takahashi K, Okita K, et al: Hypoxia enhances the generation of induced pluripotent stem cells. Cell Stem Cell 5: 237-241, 2009.

13. Hoshino H, Nagano H, Haraguchi N, et al: Hypoxia and TP53 deficiency for induced pluripotent stem cell-like properties in gastrointestinal cancer. Int J Oncol 40: 1423-1430, 2012.

14. Anokye-Danso F, Trivedi CM, Juhr D, et al: Highly efficient miRNA-mediated reprogramming of mouse and human somatic cells to pluripotency. Cell Stem Cell 8: 376-388, 2011.

15. Miyoshi N, Ishii H, Nagano H, et al: Reprogramming of mouse and human cells to pluripotency using mature microRNAs. Cell Stem Cell 8: 633-638, 2011

16. Lin SL, Chang DC, Ying SY, et al: MicroRNA miR-302 inhibits the tumorigenecity of human pluripotent stem cells by coordinate suppression of the CDK 2 and CDK4/6 cell cycle pathways. Cancer Res 70: 9473-9482, 2010.

17. Lin SL, Chang DC, Chang-Lin S, et al: Mir-302 reprograms human skin cancer cells into a pluripotent ES-cell-like state. RNA 14: 2115-2124, 2008.

18. Fareh M, Turchi L, Virolle V, et al: The miR 302-367 cluster drastically affects self-renewal and infiltration properties of glioma-initiating cells through CXCR4 repression and consequent disruption of the SHH-GLI-NANOG network. Cell Death Differ 19: 232-244, 2012

19. Dexter DL and Leith JT: Tumor heterogeneity and drug resistance. J Clin Oncol 4: 244-257, 1986.

20. Adams JM and Strasser A: Is tumor growth sustained by rare cancer stem cells or dominant clones? Cancer Res 68: 4018-4021, 2008.

21. Reya T, Morrison SJ, Clarke MF and Weissman IL: Stem cells, cancer, and cancer stem cells. Nature 414: 105-111, 2001.

22. Bonnet D and Dick JE: Human acute leukemia is organized as a hierarchy that originates from a primitive hematopoietic cell. Nat Med 3: 730-737, 1997.

23. Prince ME, Sivanandan R, Kaczorowski A, et al: Identification of a subpopulation of cells with cancer stem cell properties in head and neck squamous cell carcinoma. Proc Natl Acad Sci USA 104: 973-978, 2007.

24. Haraguchi N, Utsunomiya $\mathrm{T}$, Inoue $\mathrm{H}$, et al: Characterization of a side population of cancer cells from human gastrointestinal system. Stem Cells 24: 506-513, 2006

25. Ricci-Vitiani L, Lombardi DG, Pilozzi E, et al: Identification and expansion of human colon-cancer-initiating cells. Nature 445: 111-115, 2007.

26. O'Brien CA, Pollett A, Gallinger S and Dick JE: A human colon cancer cell capable of initiating tumour growth in immunodeficient mice. Nature 445: 106-110, 2007.

27. Al-Hajj M, Wicha MS, Benito-Hernandez A, et al: Prospective identification of tumorigenic breast cancer cells. Proc Natl Acad Sci USA 100: 3983-3988, 2003.

28. Piccirillo SG, Reynolds BA, Zanetti N, et al: Bone morphogenetic proteins inhibit the tumorigenic potential of human brain tumour-initiating cells. Nature 444: 761-765, 2006.

29. Bao S, Wu Q, McLendon RE, et al: Glioma stem cells promote radioresistance by preferential activation of the DNA damage response. Nature 444: 756-760, 2006.

30. Bixby S, Kruger GM, Mosher JT, et al: Cell-intrinsic differences between stem cells from different regions of the peripheral nervous system regulate the generation of neural diversity. Neuron 35: 643-656, 2002.

31. Sagar J, Chaib B, Sales K, et al: Role of stem cells in cancer therapy and cancer stem cells: a review. Cancer Cell Int 7: 9, 2007.

32. Zhong $\mathrm{J}$ and Dai LC: Targeting liposomal nanomedicine to cancer therapy. Technol Cancer Res Treat: Mar 28, 2012 (Epub ahead of print).

33. Hurt EM, Chan K, Serrat MA, et al: Identification of vitronectin as an extrinsic inducer of cancer stem cell differentiation and tumor formation. Stem Cells 28: 390-398, 2010.

34. Yu CH, Law JB, Suryana M, et al: Early integrin binding to Arg-Gly-Asp peptide activates actin polymerization and contractile movement that stimulates outward translocation. Proc Natl Acad Sci USA 108: 20585-20590, 2011. 